\title{
Behandlung von Schuppenflechte
}

\section{Antikörper machen Psoriasis unsichtbar}

Der monoklonale Antikörper Ixekizumab zeigt neben hohen Ansprechraten eine stabile Langzeitwirkung.

Nach der Zulassung durch die FDA im ersten Quartal 2016 ist Ixekizumab (Taltz ${ }^{\circledR}$ ) seit April nun auch in Europa zur Therapie von Erwachsenen mit mittelschwerer bis schwerer Plaque-Psoriasis zugelassen. Der humanisierte monoklonale Antikörper Ixekizumab bindet an das Zytokin Interleukin-17A (IL-17A) und neutralisiert es.

\section{Starke Wirkung}

Dafür sprechen auch die Daten aus den drei multizentrischen, doppelblinden, placebo-kontrollierten Zulassungsstudien UNCOVER 1-3 [1, 2]. Nach zwölf Behandlungswochen erreichten $90 \%$ der Patienten eine Symptomverbesserung der Haut um 75\% (PASI-75; Psoriasis Area and Severity Index). Bei rund
$70 \%$ der Teilnehmer war die Haut nahezu erscheinungsfrei (PASI-90), während etwa jeder Vierte keine Läsionen mehr zeigte (PASI-100). Die unter dem subkutan injizierten IL-17A-Inhibitor erzielten hohen Ansprechraten waren über 60 Wochen hinweg stabil.

Die Patienten erhalten nach einer Initialdosis von zweimal $80 \mathrm{mg}$ Ixekizumab über zwölf Wochen je $80 \mathrm{mg}$ Antikörper alle zwei Wochen. Danach erfolgt die Injektion alle vier Wochen in der gleichen Dosis. Häufigste Nebenwirkungen sind Reaktionen an der Injektionsstelle oder Infektionen der oberen Atemwege. Eine aktuelle Studie gibt außerdem Hinweise darauf, dass Ixekizumab bei Patienten mit Psoriasis-Arthritis zur Verbesserung der physischen Funktionalität beiträgt [3].

\section{Literatur}

1. Gordon et al (2016) Phase 3 Trials of Ixekizumab in Moderate-to-Severe Plaque Psoriasis. N Engl J Med (epub 2016 Jun 8)

2. Griffiths et al (2015) Comparison of ixekizumab with etanercept or placebo in moderate-to-severe psoriasis (UNCOVER-2 and UNCOVER-3): results from two phase 3 randomised trials. Lancet 386(9993):541-551

3. Gottlieb et al (2016) Ixekizumab Provides Improvements Through 52 Weeks in

Physical Function, Quality of Life, and Work Productivity in Biologic Disease-Modifying Antirheumatic Drug-Naïve Patients With Active Psoriatic Arthritis. [Poster] $5^{\text {th }}$ Congress of the Psoriasis international network, Paris, 07.Juli 2016: Poster 003

hautnah $2016 \cdot 15: 99$

DOI 10.1007/s12326-016-0214-4

Online publiziert: 13. Oktober 2016

(C) Springer-Verlag Wien 2016
Bericht: Julia

Rustemeier

Quelle: Globales

Pressegespräch "Lilly

in dermatology";

im Rahmen des 5th

Congress of the Pso-

riasis International

Network; 07.Juli 2016,

Paris.

Hier steht eine Anzeige. 